\title{
BMJ Open Augmented reality in patient education and health literacy: a scoping review protocol
}

To cite: Adapa K, Jain S, Kanwar R, et al. Augmented reality in patient education and health literacy: a scoping review protocol. BMJ Open 2020;10:e038416. doi:10.1136/ bmjopen-2020-038416

- Prepublication history for this paper is available online. To view these files, please visit the journal online (http://dx.doi. org/10.1136/bmjopen-2020 038416).

Received 11 March 2020 Revised 25 June 2020 Accepted 13 August 2020

\section{Check for updates}

\section{Author(s) (or their} employer(s)) 2020. Re-use permitted under CC BY-NC. No commercial re-use. See rights and permissions. Published by BMJ.

1Department of Radiation Oncology and Carolina Health Informatics Program, University of North Carolina System, Chapel Hill, North Carolina, USA ${ }^{2}$ Department of Radiation Oncology, University of North Carolina System, Chapel Hill, North Carolina, USA

${ }^{3}$ Health Sciences Library, University of North Carolina System, Chapel Hill, North Carolina, USA

Correspondence to Dr Karthik Adapa; karthikk@live.unc.edu

\section{ABSTRACT}

Background Health literacy enables the patients in understanding the basic healthcare information and taking informed health decisions; thus, it is a desirable goal of any healthcare system. It increases patients' adherence to treatment, improves the quality of care and eases the overall burden on the healthcare system. In recent years, technological solutions are being increasingly used in educating patients and achieving better health literacy. Augmented reality (AR) provides powerful, contextual and situated learning experiences and supplements the real world with virtual objects. AR could potentially be an effective learning methodology for the patients, thus, warranting a comprehensive overview of the current state of AR in patient education and health literacy.

Methods The proposed scoping review will be based on the framework developed by Arksey and 0'Malley, including the refinements suggested by Levac et al. A systematic search for references in the published literature will be conducted in nine research databasesInstitute of Electrical and Electronics Engineers (IEEE), Cumulative Index to Nursing and Allied Health Literature (CINAHL), PubMed, Psyclnfo, Embase, Web of Science, Scopus, Association for Computing Machinery (ACM) and Association for Information Systems eLibrary (AISeL). The unpublished studies from ProQuest Dissertations and Theses, Conference Proceedings Citation Index and grey literature references obtained from a web search will also be included. Databases will be searched from inception to 14 January 2020. Two independent reviewers will screen the studies from the search results in two successive stages of title/abstract screening followed by full-text screening. Data variables will be extracted from the selected studies to characterise study design, type of AR technology employed and the relational factors affecting patient education. Lastly, key stakeholders will be consulted to gather their insights about the study findings. Ethics and dissemination The results will be disseminated through stakeholder meetings and conference presentations. The data used are from publicly available secondary sources, so this study does not require ethical review.

\section{INTRODUCTION}

Patient education is a key element in the healthcare domain. When patients understand medical information communicated

\section{Strengths and limitations of this study}

- Provides a comprehensive overview of the existing research on the use of augmented reality (AR) in patient education and health literacy.

- Identifies the strengths and limitations of AR in patient education and health literacy.

- Provides an in-depth search strategy, elaborate inclusion and exclusion criteria and clear data extraction framework to address the research question.

- Quality of evidence will not be assessed, and therefore, the generalisability of the findings will not be evaluated.

by healthcare providers, they can manage their health better. ${ }^{1}$ As a result, diverse ways to educate patients regarding their health information are being explored. With the advances in health information technology (HIT), patient education has transformed from delivering simple static, text-based material to sophisticated interactive websitebased systems. ${ }^{2-5}$ For instance, a web-based patient portal enables patients in accessing their electronic health records and participating in informed decision-making. ${ }^{6}$ Similarly, virtual care clinic, another patient education tool facilitates patients in telecommunicating with the provider using video and smartphones, sometimes including telemonitoring which can collect health data remotely and non-intrusively. ${ }^{7-16}$ Further, in numerous patient care scenarios, it becomes necessary for the provider to share with the patient more complex information about the disease and facilitate a better understanding of the treatment options and rehabilitation. In these scenarios, despite all the technological advancements in HIT, presenting and comprehending such complex information remain a challenge.

To overcome the limitations in sharing and comprehending complex information, interactive technologies such as virtual reality 
(VR) and augmented reality (AR) are being increasingly used in healthcare, and especially inpatient education. ${ }^{13}$ Although these two technologies are very similar, VR engages users by transposing their virtual avatars into an artificial environment for users to interact with, while AR engages users in the real environment by overlaying virtual elements in the real world. ${ }^{17}$ Due to the immersive nature, VR presents several disadvantages to its users who may feel cyber sickness through disorientation, headache, nausea and other symptoms associated with motion sickness. ${ }^{18}$ Additionally, VR users face technological limitations in manipulating tools and components in the virtual world resulting in distraction in learning due to interference with VR. ${ }^{18-21}$ On the other hand, AR removes these limitations in learning by blending into the users' reality with minimal interference; that is, the users do not completely immerse into virtual space and can see the superimposed objects within the real-world environment. This enables the AR users to be more interactive with the task in hand.

AR enables its users to perform a variety of tasks, including displaying and manipulating information within one's field of view, mapping virtual images to real objects and video conferencing. ${ }^{22} 23$ Studies have demonstrated AR applications in multiple settings including an app-based medication management plan and medication adherence for patients, ${ }^{24} 25$ teaching anatomy to students allowing educators to deviate from a traditional classroom setting, ${ }^{22}$ simulating objects aids in surgery to better visualise organs ${ }^{22} 2627$ and improving tracking technique in rehabilitation for better physical outcomes. ${ }^{28}{ }^{29}$ Thus, the AR systems support visualising invisible or abstract concepts and promoting conceptual understanding of dynamic models and complex causality. ${ }^{30} 31$

Many systematic and scoping reviews have been conducted in AR. However, most of these reviews focused on applications of AR in broad fields such as medicine ${ }^{32}$ and nursing ${ }^{33}$ or specialised fields such as surgery, ${ }^{34}$ neurosurgery, ${ }^{27}$ otolaryngology, ${ }^{35}$ dentistry, ${ }^{36}$ emergency medicine ${ }^{22}$ and so on. Sherstyuk et al reviewed the current state of mixed reality manikins for medical education. ${ }^{37}$ Similarly, Zhu et al reviewed the effect of AR in developing competencies in healthcare but they focused on the education of healthcare professionals. ${ }^{29}$ These reviews helped in understanding the acceptance of AR as a learning technology in healthcare and its potential for improving different types of competencies. However, to date, no study has focused exclusively on patients or reviewed applications of $\mathrm{AR}$ in patient education and health literacy.

AR has immense potential in providing an effective learning and training methodology for communicating critical but complex information. Previous reviews have evaluated the use of AR in healthcare education but have not focused on using AR for patient education and health literacy. Therefore, the objective of this paper is to present a protocol for carrying out a scoping review. The scoping review will provide a comprehensive overview of the existing research on the use of AR in patient education and health literacy. Through the scoping review, we aim to identify research gaps, strengths and limitations of $\mathrm{AR}$ in patient education and health literacy and provide insights to guide future research.

\section{METHODS}

We will conduct a scoping review as described by Arksey and O'Malley. ${ }^{38}$ A scoping review involves knowledge synthesis and addresses an exploratory research question aimed at mapping key concepts (elements), types of evidence and gaps in research. ${ }^{39}$ Scoping studies are particularly relevant in disciplines with emerging evidence such as AR in patient education and health literacy that have not been comprehensively reviewed previously. ${ }^{40}$ These studies involve systematic searches for, and selection and synthesis of, existing knowledge across a range of study designs. ${ }^{39}{ }^{41}$ However, unlike a systematic review, scoping studies typically do not require weighing the evidence using a formal assessment of methodological quality. We are publishing this protocol per the Preferred Reporting Items for Systematic Review and Meta-Analysis Extension for Scoping Reviews (PRISMA-ScR) statement. ${ }^{42}$ We want to highlight that while reporting guidelines are defined for protocols of systematic reviews, ${ }^{434}$ there are currently no corresponding reporting guidelines for protocols of scoping reviews. Therefore, we have reasonably adopted the PRISMA-ScR guidelines as applicable to the protocol stage of publication (attached as a online supplementary file).

This review protocol proposes a methodological approach to the scoping review before carrying out the review. Review protocols allow reviewers to carefully plan and thereby anticipate potential problems and avoid arbitrary decision-making when conducting a review. Protocols also enable others to identify selective reporting, replicate methods and judge the validity of proposed methods. Further, the publication of protocols reduces the duplication of reviews on an identical topic and prompts potential future collaborations. ${ }^{43}$ While there is not a universally accepted terminology, definition or methodology for scoping reviews, the design of this protocol is based on the framework by Arksey and $\mathrm{O}^{\prime}$ Malley ${ }^{38}$ and the refinements to this work by Levac et $a l^{40}$ We have accordingly organised this protocol into six stages, which are explained below.

\section{Stage 1: identification of the research question}

In this stage, there are two primary tasks: (1) identifying a broad research question that serves as a roadmap for subsequent stages and (2) establishing the scope of inquiry which includes defining the concept, identifying the target population and the outcomes of interest. Accomplishing these tasks would assist in the identification and selection of studies in subsequent stages. We undertake this review to answer the following research question: 
Table 1 Definitions of key concepts within our research question

\begin{tabular}{ll} 
Concept & Definition \\
\hline $\begin{array}{l}\text { Augmented reality } \\
\text { (AR) }\end{array}$ & $\begin{array}{l}\text { AR is a technology that allows a live real-time direct or indirect real-world environment to be enhanced } \\
\text { by computer-generated virtual imagery information } 5354\end{array}$ \\
Patient education & $\begin{array}{l}\text { "Procedure in which individuals with health occupations impart information to patients about their own } \\
\text { health status and needs. Education can be therapeutic or used for disease prevention"55 }\end{array}$ \\
Health literacy & $\begin{array}{l}\text { "Degree to which an individual has the capacity to obtain, communicate, process, and understand basic } \\
\text { health information and services to make appropriate health decisions"56 }\end{array}$ \\
\hline
\end{tabular}

'To date, what research has been performed regarding the use of AR in patient education and health literacy?'

Key concepts within our research question include 'augmented reality (AR)', 'patient education' and 'health literacy'. We define these concepts in table 1

Patient education and health literacy are related but distinct concepts, being two separate sides of the same coin. The principal goal of patient education is to instill a sense of autonomy in the patients and provide them with the knowledge required for self-management and taking healthcare decisions. ${ }^{45} 46$ Effective patient education efforts are likely to increase the frequency of shared decision-making. ${ }^{47}$ Patients successfully educated by providers can better understand their health and interact effectively with healthcare providers. ${ }^{48}$ These interactions encompass finding information and services, communicating their needs and preferences, and understanding the choices, consequences, and context of information and services. ${ }^{149}$ This enables them to subsequently decide which information and services match their needs and preferences so that they can take necessary action. ${ }^{46}$ Thus, health literacy is the end goal of strong patient education. $^{4550}$

The population of interest for this scoping review includes all studies using AR for patient education or health literacy. The envisioned outcome is a description of the evidence supporting AR in patient education and health literacy, as well as a descriptive summary of the studies that were identified; this descriptive summary will help discover the research gaps of using AR in patient education and health literacy and assess the strengths and limitations of AR in patient education and health literacy.

The context for this scoping review is broadly defined and shall include studies conducted in all settings, all geographical locations, and all types of study designs.

\section{Stage 2: identification of relevant studies}

This stage involves balancing the breadth and depth of the scoping review with feasibility to conduct the review.

\section{Search methods}

To answer the research question, we will conduct a systematic search of the following nine electronic databases from inception to 14 January 2020: Instituteof Electrical and Electronics Engineers (IEEE), CumulativeIndex to Nursing and Allied Health Literature (CINAHL), PubMed, PsycInfo, Embase, Web of Science, Scopus,
Association for Computing Machinery (ACM) and Associationfor Information Systems eLibrary (AISeL). The selection of these databases from the field of healthcare, informatics and related cross-functional disciplines has been made given the interdisciplinary nature of the research questions.

We will use a comprehensive search strategy that uses search terms (keywords and/or medical subject headings or subject headings) that relate to key concepts, combines search terms within a concept with the Boolean term 'OR', combines search terms between concepts with the Boolean term 'AND' and is adapted to the syntax used by each database. We will collaborate with an expert librarian (JW) to develop the search strategy. We present a sample search strategy for PubMed in table 2. A preliminary search on PubMed yielded 63 results. The first group was 'augmented reality' and included terminology with similar meaning such as 'mixed reality' or 'blended reality'. The second group was patient education and health literacy and included terms such as medication knowledge, medication literacy, e-health literacy and digital health literacy.

The search strategy was modified for IEEE and AISEL databases which had restrictions in the number of search terms or did not use proximity operator 'NEAR' in some form. We will additionally search for unpublished studies through an electronic database (ie, ProQuest Dissertations \& Theses, Conference Proceedings Citation Index) and website (Google) searches. When searching in Google, we will use a more specific search string query, such as "Augmented reality for patient education," "Augmented reality for health literacy," "Augmented reality for patient engagement," and "Augmented reality for medical literacy." We will analyse the top 100 results in Google to identify studies. We will follow-up search of electronic databases and websites with snowball sampling. ${ }^{51}$ This includes backward and forward reference searching for included articles and correspondence with colleagues and experts in the field about augmented reality, patient education, health literacy, patient engagement and medication knowledge. Searches will be conducted in each database with no date restriction, no methodological filter and the language limited to English. We will use EndNote, a reference manager tool to compile relevant literature and to identify duplicates. 


\begin{tabular}{|c|c|c|}
\hline Search & Query & $\begin{array}{l}\text { Items } \\
\text { found }\end{array}$ \\
\hline 1 & (augmented reality OR mixed reality OR blended reality) & 3286 \\
\hline
\end{tabular}

\section{Inclusion/exclusion criteria}

To present a comprehensive overview of AR in patient education and health literacy, we will use broad inclusion criteria as outlined in table 3 . An article will be included in the review if it is a research paper on AR with a study focus exclusively on patients (patient education or patient engagement or medication knowledge or health literacy in patients) and written in English. We will exclude articles that are unavailable as full texts, published in a non-English language, and those due to feasibility relatedreasons (eg, limited resources, including funding to hire translators).

\section{Stage 3: study selection}

We will use the following process for study selection, using the inclusion/exclusion criteria identified above.

1. The expert librarian, JW, will implement the search strategy and retrieve the references obtained from published literature search using the nine databases

\section{Table 3 Inclusion and exclusion criteria}

\begin{tabular}{|c|c|c|}
\hline Parameter & Inclusion criteria & Exclusion criteria \\
\hline Language & English & Non-English \\
\hline Study focus & $\begin{array}{l}\text { Any study } \\
\text { focused on the } \\
\text { use of } A R \text { in } \\
\text { patient education } \\
\text { and health } \\
\text { literacy }\end{array}$ & $\begin{array}{l}\text { Any study not using } \\
\text { AR } \\
\text { Any study not } \\
\text { focused on patients } \\
\text { Any study not } \\
\text { focused on health } \\
\text { literacy }\end{array}$ \\
\hline Availability & Full text available & $\begin{array}{l}\text { Full text not } \\
\text { available }\end{array}$ \\
\hline Study design & Any & $\mathrm{Nil}$ \\
\hline Setting & Any & $\mathrm{Nil}$ \\
\hline
\end{tabular}

AR, augmented reality. identified above, and from the unpublished literature search using ProQuest, Conference proceedings citation index and website search.

2. Before commencing the screening process, we will conduct a calibration exercise to ensure reliability in correctly selecting articles for inclusion. It will entail independently screening a random sample of $5 \%$ of the included citations by SK and RK. Eligibility criteria will be modified if a low agreement is observed between the reviewers (eg, a kappa statistic less than $50 \%$ ). The reviewers will then independently screen the remainder of the search results using a predefined relevance criterion form for all levels of screening (eg, title and abstract, full-text review). Discrepancies will be resolved independently by KA.

3. SJ and RK will then take the screened references which have been selected from the previous step, and conduct further screening based on the full text of the references. They will record their decision whether an article is included or excluded and the primary reason for exclusion, where applicable. As before, any conflicts in these screening decisions will be resolved by KA.

4. SJ and RK will take the included references from the previous stage as seeding point for snowball sampling using backward and forward reference searching and taking inputs from experts and colleagues, JW and LM. For snowball sampling, JW will conduct a followup search of electronic databases and websites. The results from this follow-up search will be screened again using the process outlined in steps 2 and 3 above. The new set of included references will be combined with the initial set of included references, to get a final set of selected studies.

We will use Covidence (Veritas Health Innovation, Melbourne, Australia) to manage study selection. Covidence is an online application designed to support the 


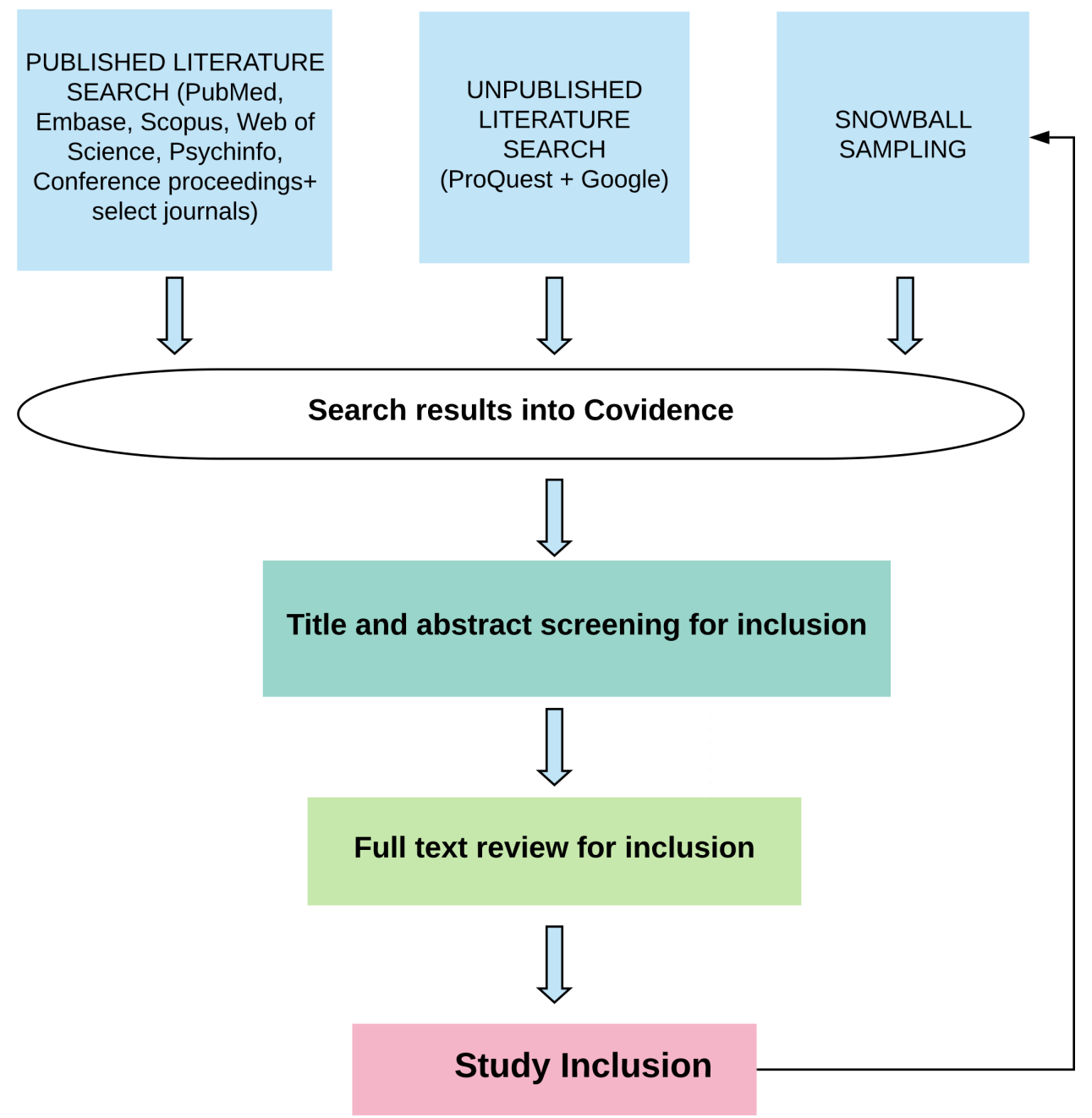

Figure 1 Flow of studies within stages 2 and 3.

production and management of systematic reviews. ${ }^{52}$ It is the standard production platform for Cochrane Reviews and can be used for scoping studies. A project will be created in Covidence with KA, SJ, RK, JW and LM as members. Figure 1 displays the flow of studies within stages 2 and 3 .

\section{Stage 4: extracting the data}

SJ and RK will pilot test a data extraction form on a random sample of 2-3 included full texts of articles and revise it iteratively. The differences in data extraction will be resolved by discussion with a third reviewer (KA). To classify the publications, we will choose classification methods to generate a comparable dataset within the research of AR in patient education and health literacy. The three main characteristics, including research, $\mathrm{AR}$ and patient education from the included studies, and the 21 subcharacteristics will be described through qualitative (eg, content analysis) and quantitative (eg, frequency analysis, mean, etc) methods, as outlined in table 4 . Thematic analysis will also be used to identify the prominent themes that describe the current use of AR in patient education and health literacy. These themes will then be presented in the Results section in terms of the strengths and weaknesses of $\mathrm{AR}$ in patient education and health literacy.

\section{Stage 5: collating, summarising and reporting the results}

This stage involves considerations related to data analysis, reporting of results and implications of findings. Our primary objective is to provide an overview of the existing research on the use of AR in patient education and health literacy.

We will provide a descriptive analysis encompassing the following characteristics of the included articles:

- research study characteristics (study type, study objective, methods used, use case description, evaluation),

- technology characteristics (research maturity, AR display technology, and challenges, AR tracking, AR taxonomy, interactive or haptic), and

- patient education characteristics (learning theory, learning principle/strategy and learning outcomes).

We will also present a narrative analysis of the data and conduct a thematic study of the reviewed literature to highlight strengths and limitations, gaps and opportunities.

We envision that these findings will have implications for research and practice, as well as patients and researchers that choose to engage in research as partners. 
Table 4 Overview of the variables and the related classification method

\begin{tabular}{|c|c|c|}
\hline Category & Variable & Classification method \\
\hline \multicolumn{3}{|l|}{ Research } \\
\hline & Year of publication & Metadata \\
\hline & Geolocation & Metadata \\
\hline & Number of participants & Metadata \\
\hline & Participant age group & Metadata \\
\hline & Medical subject headings & $\mathrm{MeSH}^{57}$ \\
\hline & Clinical trial & Clinical trials.gov, ${ }^{58}$ manual, binary \\
\hline & Use cases identification & Manual, binary \\
\hline & Evaluation & Manual, binary \\
\hline \multicolumn{3}{|c|}{ AR technology } \\
\hline & Technology readiness level & US Department of Defense,$^{59}$ manual \\
\hline & AR display category & Schmalstieg and Höllerer, ${ }^{60}$ manual \\
\hline & Technical challenges & Schmalstieg and Höllerer ${ }^{60}$ manual \\
\hline & AR tracking & Schmalstieg and Höllerer, ${ }^{60}$ manual \\
\hline & AR taxonomy & Hugues et al, ${ }^{53}$ manual \\
\hline & Interactive or haptic & Manual, binary \\
\hline \multicolumn{3}{|c|}{ Patient education } \\
\hline & Care path & Elsevier's conceptual framework to develop patient education content ${ }^{61}$ \\
\hline & Educator & Miller-Stoeckel Client Education model, ${ }^{62}$ manual \\
\hline & Learner & Miller-Stoeckel Client Education model ${ }^{62}$ manual \\
\hline & Educator-learner relationship & Miller-Stoeckel Client Education model, ${ }^{62}$ manual \\
\hline & Patient education outcomes & Miller-Stoeckel Client Education model, ${ }^{62}$ manual \\
\hline & Learning theory & Manual, binary \\
\hline & Learning strategy & Manual \\
\hline & Learning styles & Manual \\
\hline & Teaching techniques & Manual \\
\hline
\end{tabular}

$A R$, augmented reality.

For example, this review will highlight the research gaps in using AR for patient education and health literacy, provide evidence regarding the use of AR to improve patient learning and the design principles that have been considered for improving patient satisfaction and can be used to guide future research. It will also identify the strengths and weaknesses of using AR in patient education and health literacy. This could serve as a solid starting point for understanding applications of AR in patient education, and health literacy.

\section{Stage 6: consultation}

In Arksey and O'Malley's recommended framework for conducting scoping reviews, ${ }^{38}$ consultation is an optional step. However, Levac et $a \vec{l}^{39}{ }^{40}$ consider a consultation as a critical component of their scoping review methodology. The goal of the consultation exercise is to take the findings from the scoping review to the population it is expected to benefit, namely the patients, and to contextualise the findings based on the experiences of the patients. According to Levac et al, it is only after successful consultation that the research team can validate their findings. Consultation is a knowledge translation exercise, in that it ensures that the design, conduct and dissemination of knowledge arising from the scoping review remain relevant not only to the research community but also to the patients.

Accordingly, we propose to consult a diverse group of stakeholders including two patients who have participated in at least one research project related to patient education using augmented reality. The primary method of engagement will be direct interactions, such as interviews or small-group meetings for sharing of information and their experiences. We will conduct these interactions in a semistructured, semiformal fashion to encourage patients to open up about their ideas and reflections on their participation.

Another aspect of this stage in the research process is that it provides an opportunity to plan the dissemination of the findings to the community through the most important stakeholders in this research. To facilitate an 
Table 5 Overview of the terms of reference

\begin{tabular}{|c|c|}
\hline Subsection & Key components, including examples \\
\hline Preamble & Background and project objectives \\
\hline Research team & Team members' names, positions, roles and responsibilities \\
\hline $\begin{array}{l}\text { Responsibilities and opportunities for patient } \\
\text { partners }\end{array}$ & $\begin{array}{l}\text { For example, to partner with the research team to participate in the } \\
\text { consultation activity, share feedback, perspectives and experiences, be } \\
\text { available during consultations }\end{array}$ \\
\hline General expectations related to patient engagement & $\begin{array}{l}\text { For example, to recognise lived experience as a form of knowledge and } \\
\text { expertise, be mindful of wording for any written materials, maintain a fair } \\
\text { and structured relationship that does not cross professional boundaries } \\
\text { and so on. }\end{array}$ \\
\hline Contingencies & Assumptions, known constraints, estimated risks, mitigation plan \\
\hline Expected outcomes & $\begin{array}{l}\text { Major project milestones and deliverable, for example, proposed } \\
\text { manuscripts, presentations, potential future projects and so on. }\end{array}$ \\
\hline Criteria for success & $\begin{array}{l}\text { Measurable success metrics, for example, approval by more than } 70 \% \text { of } \\
\text { stakeholders }\end{array}$ \\
\hline
\end{tabular}

active collaboration between the research team and the stakeholders, the research team (KA, SJ and RK) shall develop a terms of reference document. This document shall provide an overview of the project, identify the names and positions of engaged research team members, and the nature of the relationships and expectations between patient partners and researchers. We expand on the subsections of this document in table 5 .

\section{Patient and public involvement}

There was no patient or public involvement in the design of this protocol.

\section{DISCUSSION}

As a new and emerging topic, AR has found applications primarily on training healthcare providers, rather than focusing on patients. AR has shown potential as a teaching tool for medically trained and untrained individuals. By natural extension, it holds promise as a robust teaching tool for patients as well.

To the best of our knowledge, this is the first scoping review on the use of $A R$ in health literacy and patient education. To that effect, we aim to characterize the nature and extent of evidence by assessing the study design, type of AR technology employed, and the relational factors affecting patient education. Due to the variable nature of conducting scoping reviews, the focus of this paper has been to elucidate the methodology used to guide future research in the domain.

\section{Strengths and limitations of this study}

This review may serve as an informational tool to standardise prospective scoping reviews in the field of healthcare education and AR. The protocol outlined above is intended to be rigorous, transparent and consistent with the framework established by Arksey and O'Malley. Also, we worked with an expert librarian to devise the search strategy to be as compendious as possible. However, due to the wide scope of this study approach, a common limitation may be the inability to retrieve and review all available literature. While we strived to be comprehensive by conducting a systematic search across the nine largest healthcare and informatics databases, our review is not purported to be exhaustive.

\section{Dissemination and ethics}

Since this article does not involve human subjects, approval from the Institutional Review Board was not required. This paper will be submitted for publication in a peer-reviewed scientific journal and presented at relevant conferences. The steps outlined in this paper are anticipated to be useful for new and established researchers interested in conducting scoping reviews. It will also guide health professionals interested in planning, organising and conducting future research in the application of augmented reality technologies for patient education and health literacy.

Acknowledgements The authors acknowledge the support of Department of Radiation Oncology, UNC-Chapel Hill and the Health Sciences Library, UNC.

Contributors KA conceived the study, outlined the protocol, and is the guarantor of the review. KA, SJ, TZ and TT jointly developed the research question and wrote the 
first draft of the manuscript with responsibilities for single sections. JW developed the methodology for this study, devised the search strategy and conducted the final decision for inclusion and exclusion criteria and the databases to be included for this study. RK revised and edited the paper and made a substantial contribution to the final draft of the manuscript. LM supervised the entire research and mentored the team. All authors further revised the paper and approved the final manuscript.

Funding The authors have not declared a specific grant for this research from any funding agency in the public, commercial or not-for-profit sectors.

Competing interests None declared.

Patient and public involvement Patients and/or the public were not involved in the design, or conduct, or reporting, or dissemination plans of this research.

Patient consent for publication Not required.

Provenance and peer review Not commissioned; externally peer reviewed.

Open access This is an open access article distributed in accordance with the Creative Commons Attribution Non Commercial (CC BY-NC 4.0) license, which permits others to distribute, remix, adapt, build upon this work non-commercially, and license their derivative works on different terms, provided the original work is properly cited, appropriate credit is given, any changes made indicated, and the use is non-commercial. See: http://creativecommons.org/licenses/by-nc/4.0/.

ORCID iD

Karthik Adapa http://orcid.org/0000-0002-3970-588X

\section{REFERENCES}

1 Marcus C. Strategies for improving the quality of verbal patient and family education: a review of the literature and creation of the educate model. Health Psychol Behav Med 2014;2:482-95.

2 Richards B, Colman AW, Hollingsworth RA. The current and future role of the Internet in patient education. Int $J$ Med Inform 1998;50:279-85.

3 Prey JE, Woollen J, Wilcox L, et al. Patient engagement in the inpatient setting: a systematic review. J Am Med Inform Assoc 2014;21:742-50.

4 Eng TR. The eHealth landscape: a terrain map of emerging information and communication technologies in health and health care 2001.

5 Oh H, Rizo C, Enkin M, et al. What is eHealth (3): a systematic review of published definitions. J Med Internet Res 2005;7:e1.

6 Foster B, Krasowski MD. The use of an electronic health record patient portal to access diagnostic test results by emergency patients at an academic medical center: retrospective study. J Med Internet Res 2019;21:e13791.

7 Dorsey ER, Topol EJ. State of telehealth. N Engl J Med 2016;375:154-61.

8 van Dyk L. A review of telehealth service implementation frameworks. Int J Environ Res Public Health 2014;11:1279-98.

9 Warmington K, Flewelling C, Kennedy CA, et al. Telemedicine delivery of patient education in remote Ontario communities: feasibility of an Advanced Clinician Practitioner in Arthritis Care (ACPAC)-led inflammatory arthritis education program. Open Access Rheumatol 2017;9:11-19.

10 Conde JG, De S, Hall RW, et al. Telehealth innovations in health education and training. Telemed J E Health 2010;16:103-6.

11 Tomlinson M, Solomon W, Singh Y, et al. The use of mobile phones as a data collection tool: a report from a household survey in South Africa. BMC Med Inform Decis Mak 2009;9:51.

12 van Heerden A, Tomlinson M, Swartz L. Point of care in your pocket: a research agenda for the field of $\mathrm{m}$-health. Bull World Health Organ 2012;90:393-4.

13 Hsieh MC, Lee JJ. Preliminary study of VR and Ar applications in medical and healthcare education. J Nurs Health Stud 2018;03.

14 Tomlinson M, Rotheram-Borus MJ, Swartz L, et al. Scaling up mHealth: where is the evidence? PLoS Med 2013;10:e1001382.

15 Rotheram-Borus MJ, le Roux IM, Tomlinson M, et al. Philani plus (+): a mentor mother community health worker home visiting program to improve maternal and infants' outcomes. Prev Sci 2011;12:372-88.

16 Siedner MJ, Haberer JE, Bwana MB, et al. High acceptability for cell phone text messages to improve communication of laboratory results with HIV-infected patients in rural Uganda: a cross-sectional survey study. BMC Med Inform Decis Mak 2012;12:56.

17 Herron J. Augmented reality in medical education and training. $J$ Electron Resour Medical Libr 2016;13:51-5.
18 Valimont B. The effectiveness of an augmented reality learning paradigm 2002.

19 Cutmore TIMRH, Hine TJ, Maberly KJ, et al. Cognitive and gender factors influencing navigation in a virtual environment. Int J Hum Comput Stud 2000;53:223-49.

20 Barnett B, Helbing K, Hancock G, et al. An evaluation of the training effectiveness of virtual environments 2000.

21 Kennedy RS, Stanney KM. Aftereffects of virtual environment exposure: psychometric issues. Advances in human factors/ ergonomics 1997.

22 Munzer BW, Khan MM, Shipman B, et al. Augmented reality in emergency medicine: a scoping review. J Med Internet Res 2019;21:e12368

23 Brigham TJ. Reality check: basics of augmented, virtual, and mixed reality. Med Ref Serv Q 2017;36:171-8.

24 Ingeson M, Blusi M, Nieves JC, et al. Microsoft Hololens - A mHealth Solution for Medication Adherence. In: Koch F, Koster A Riaño D, et al, eds. Artificial Intelligence in Health: First International Workshop, AlH 2018, Stockholm, Sweden, July 13-14, 2018, Revised Selected Papers. Cham: Springer International Publishing, 2019: 99-115.

25 Ingeson M, Blusi M, Nieves JC. Smart augmented reality mHealth for medication adherence 2018:157-68.

26 Shuhaiber JH. Augmented reality in surgery. Arch Surg 2004:139:170-4.

27 Guha D, Alotaibi NM, Nguyen N, et al. Augmented reality in neurosurgery: a review of current concepts and emerging applications. Can J Neurol Sci 2017;44:235-45.

28 Rabbi I, Ullah S, Khan SU. Augmented reality tracking techniques - a systematic literature. Journal of Computer Engineering 2012;2:23-9.

29 Zhu E, Hadadgar A, Masiello I, et al. Augmented reality in healthcare education: an integrative review. PeerJ 2014;2:e469.

$30 \mathrm{H}-\mathrm{K} \mathrm{W}$, SW-Y L, Chang H-Y, et al. Current status, opportunities and challenges of augmented reality in education. Comput Educ 2013;62:41-9.

31 Kamphuis C, Barsom E, Schijven M, et al. Augmented reality in medical education? Perspect Med Educ 2014;3:300-11.

32 Eckert M, Volmerg JS, Friedrich CM. Augmented reality in medicine: systematic and bibliographic review. JMIR Mhealth Uhealth 2019;7:e10967

33 Wüller $\mathrm{H}$, Behrens J, Garthaus $\mathrm{M}$, et al. A scoping review of augmented reality in nursing. BMC Nurs 2019;18:19.

34 Wong K, Yee HM, Xavier BA, et al. Applications of augmented reality in otolaryngology: a systematic review. Otolaryngol Head Neck Surg 2018;159:956-67.

35 Yoon JW, Chen RE, Kim EJ, et al. Augmented reality for the surgeon: systematic review. Int J Med Robot 2018;14:e1914.

36 Joda T, Gallucci GO, Wismeijer D, et al. Augmented and virtual reality in dental medicine: a systematic review. Comput Biol Med 2019;108:93-100.

37 Sherstyuk A, Vincent D, Berg B, et al. Mixed reality manikins for medical education. In: Furht B, ed. Handbook of augmented reality. New York, NY: Springer New York, 2011: 479-500.

38 Arksey H, O'Malley L. Scoping studies: towards a methodological framework. Int J Soc Res Methodol 2005;8:19-32.

39 Colquhoun HL, Levac D, O'Brien KK, et al. Scoping reviews: time for clarity in definition, methods, and reporting. J Clin Epidemiol 2014;67:1291-4

40 Levac D, Colquhoun H, O'Brien KK. Scoping studies: advancing the methodology. Implement Sci 2010;5:69.

41 Peters MDJ, Godfrey CM, Khalil H, et al. Guidance for conducting systematic scoping reviews. Int J Evid Based Healthc 2015;13:141-6.

42 Tricco AC, Lillie E, Zarin W, et al. PRISMA extension for scoping reviews (PRISMA-ScR): checklist and explanation. Ann Intern Med 2018;169:467-73.

43 Shamseer L, Moher D, Clarke M, et al. Preferred reporting items for systematic review and meta-analysis protocols (PRISMA-P) 2015: elaboration and explanation. BMJ 2015;350:g7647.

44 Moher D, Shamseer L, Clarke M, et al. Preferred reporting items for systematic review and meta-analysis protocols (PRISMA-P) 2015 statement. Syst Rev 2015;4:1.

45 Wittink H, Oosterhaven J. Patient education and health literacy. Musculoskelet Sci Pract 2018;38:120-7.

46 Heath S. The difference between patient education and health literacy. patient engagement hit, 2017. Available: https:// patientengagementhit.com/news/the-difference-between-patienteducation-and-health-literacy [Accessed 17 Dec 2019].

47 Sudore RL, Boscardin J, Feuz MA, et al. Effect of the prepare website vs an Easy-to-Read advance directive on advance care planning documentation and engagement among veterans: a randomized clinical trial. JAMA Intern Med 2017;177:1102-9. 
48 Paterick TE, Patel N, Tajik AJ, et al. Improving health outcomes through patient education and partnerships with patients. Proc 2017;30:112-3

49 Behar-Horenstein LS, Guin P, Gamble K, et al. Improving patient care through patient-family education programs. Hosp Top 2005;83:21-7.

50 Why Patient Education \& Health Literacy Is Important - BoardVitals Blog. Available: https://www.boardvitals.com/blog/patient-educationhealth-literacy/ [Accessed 15 Aug 2019].

51 Damschroder LJ, Aron DC, Keith RE, et al. Fostering implementation of health services research findings into practice: a consolidated framework for advancing implementation science. Implement Sci 2009; $4: 50$.

52 Covidence - Better systematic review management. Available: https://www.covidence.org/home [Accessed 22 Jul 2019].

53 Furht B. Handbook of Augmented Reality. New York, NY: Springer New York, 2011

54 Lee K. Augmented reality in education and training. TechTrends 2012;56:13-21.

55 Patient education - Latest research and news | Nature. Available: https://www.nature.com/subjects/patient-education [Accessed 18 Dec2019].
56 What is health literacy? | health literacy | CDC. Available: https://www. cdc.gov/healthliteracy/learn/index.html [Accessed 18 Dec 2019].

57 Nih us national library of medicine medical subject Headings. Available: https://www.nlm.nih.gov/mesh/meshhome.html [Accessed 17 Dec 2019].

58 Nih us national library of medicine clinical trials database. Available: https://www.clinicaltrials.gov/ [Accessed 17 Dec 2019].

59 Assistant Secretary of Defense for Research and Engineering (ASD(R\&E)). Technology readiness assessment (Tra) guidance. Washington, DC: United States Department of Defense, 2011. https://apps.dtic.mil/dtic/tr/fulltext/u2/a554900.pdf

60 Schmalstieg D, Höllerer T. Augmented reality: principles and practice. 1st ed.. Boston: Addison-Wesley Professional, 2016.

61 Lauren J. The patient journey: a conceptual framework for engaging patients, 2017. Available: https://www.healthleadersmedia.com/ strategy/patient-journey-conceptual-framework-engaging-patients [Accessed 30 Jul 2019].

62 Miller MA, Stoeckel PR. Client education: theory and practice. 1st edn. Sudbury, Mass: Jones \& Barlett Learning, 2010. 